\title{
CRITERIOS Y FRECUENCIA DE USO DE ESTRATEGIAS DE RETROALIMENTACIÓN CORRECTIVA: UN ESTUDIO DE CASO SOBRE LAS PERCEPCIONES DE ESTUDIANTES Y PROFESORES UNIVERSITARIOS ${ }^{1}$
}

\author{
FREQUENCY AND CRITERIA FOR USING CORRECTIVE \\ FEEDBACK: A CASE STUDY ON UNIVERSITY STUDENTS' AND \\ LECTURERS' PERCEPTIONS
}

\author{
ROXANA ORREGO \\ Universidad de Santiago de Chile \\ roxana.orrego@usach.cl \\ NÉSTOR SINGER \\ Universidad de Santiago de Chile \\ nestor.singerc@usach.cl \\ RICARDO ÚBEDA \\ Universidad de Santiago de Chile \\ ricardo.ubeda@usach.cl \\ SIXTO YÁÑEZ \\ Universidad de Santiago de Chile \\ sixtoyp@yahoo.es
}

\section{RESUMEN}

Este estudio explora las percepciones de estudiantes y profesores de un programa de Pedagogía en Inglés acerca del grado de conocimiento, criterios y frecuencia de uso de técnicas de retroalimentación correctiva por parte de profesores del área de lengua inglesa para el tratamiento de errores gramaticales propios de este contexto. Esta investigación se basa en el análisis de resultados de estudios que han examinado los tipos de retroalimentación correctiva recibida por aprendices de una segunda lengua (L2) (Lyster y Ranta, 1997; Sheen, 2004), el grado de atención a este y el nivel de asimilación (uptake) (Mackey, et al., 2003; Philp, 2003), y la caracterización del concepto 'retroalimentación

${ }^{1}$ Investigación adscrita al proyecto DICYT-USACH 031651OR denominado El impacto de la retroalimentación interaccional correctiva conocida como "recast" en el aprendizaje de estructuras gramaticales en lengua inglesa en discurso oral. 
correctiva' como la respuesta sistemática a enunciados erróneos, ya sea en forma de una intervención implícita o explícita (Ellis, Loewen y Erlam, R, 2006; Pawlak, 2014). Para llevar a cabo esta investigación se ocuparon dos métodos de recolección de datos: una encuesta y una entrevista semiestructurada aplicada a grupos focales. La primera se aplicó a cuatro niveles de una Pedagogía en Inglés y a los profesores de asignaturas de lengua inglesa, y la segunda para ahondar en dichas representaciones. Los resultados preliminares evidencian un nivel de congruencia entre los tipos de correctivos utilizados y la percepción de su pertinencia por parte de los alumnos. No obstante, los datos obtenidos reflejan que los alumnos de últimos niveles y algunos profesores poseen representaciones propias de la retroalimentación que no se condicen con lo descrito en la literatura. Se concluye que estos hallazgos apoyan la idea de que es necesario plantear cursos de perfeccionamiento especializado en estrategias y metodologías para retroalimentación correctiva en enseñanza-aprendizaje de una L2.

Palabras clave: Representaciones sociales, retroalimentación correctiva, adquisición en L2, conocimiento implícito y explícito, discurso oral en L2.

\section{ABSTRACT}

This study explores students' and teachers' perceptions towards corrective feedback knowledge, criteria and frequency of use performed by teachers to address grammar errors in English language course units. This research is based on the result analysis of previous studies that have examined types of corrective feedback received by second language (SL) learners (Lyster y Ranta,1997; Sheen, 2004), the extent of noticing it and its uptake Mackey, et al. (2003); Philp (2003), and the conceptualisation of the corrective feedback as a systematic answer to wrong utterances, either implicitly or explicitly (Ellis, R., Loewen, S. y Erlam, R., 2006; Pawlak, 2014). Two data collection methods were used to carry out this research, i.e. a survey and a semi structured interview delivered in focus groups. The first was applied to four levels of learners of an English language teaching programme and the teachers teaching English language of the same programme. Then a semi structured interview was conducted to delve into those representations. Preliminary results show students recognise there is certain coherence between the types of correctives used and their appropriateness. However, data reflect that fifth-year learners and some teachers hold their own representations towards corrective feedback which are inconsistent with the available literature. It is concluded that these findings support the claim that teacher development programmes on SL corrective feedback strategies and methodologies are necessary.

Keywords: Social representations, corrective feedback, SL acquisition, implicit and explicit knowledge, oral discourse in SL.

Recibido: 24/06/2018. Aceptado: 30/05/2019. 


\section{INTRODUCCIÓN}

Zl objetivo central de este estudio radica en la exploración y análisis de las Cpercepciones de profesores y estudiantes de un programa de Pedagogía en Inglés en cuanto a (i) el grado de conocimiento teórico y práctico de técnicas de retroalimentación correctiva, (ii) los criterios pedagógicos que determinan su uso y (iii) la frecuencia de uso de estas en el contexto de los cursos que conforman la línea de lengua inglesa de dicho programa.

Una aproximación descriptiva a esta problemática presupone la caracterización de la retroalimentación correctiva como una alternativa de intervención pedagógica en forma de una respuesta sistemática a enunciados que contienen una o más formas erróneas producidos por aprendices de una segunda lengua (L2). Del mismo modo, también considera el grado de atención a este por parte de los aprendices en un contexto de instrucción formal, y el potencial nivel de asimilación de la evidencia presentada a través de la instancia de retroalimentación correctiva: uptake.

En este contexto, un amplio número de enfoques psicolingüísticos que caracterizan el proceso de adquisición de una segunda lengua (L2) parecen encontrar en el grado de explicitación de la retroalimentación correctiva el sustento teórico que informa las decisiones pedagógicas de los profesores de lengua en cuanto a (i) el tipo de retroalimentación correctiva y su frecuencia de uso, (ii) la optimización de la evidencia presentada a través de la retroalimentación correctiva para optimizar las condiciones que faciliten los procesos cognitivos de atención a esta y a la interpretación de su intención correctiva por parte de los aprendices y (iii) un factor relevante para la medición empírica del efecto de la retroalimentación correctiva en términos del grado de asimilación de la evidencia presentada por parte de los aprendices.

Así, una medición global y efectiva del grado de contribución de la retroalimentación correctiva al proceso de adquisición de una segunda lengua (L2) que complemente la evidencia empírica obtenida a través de estudios experimentales iniciales Ammar y Spada (2006); Ayoun (2004); Han (2002); Leeman (2003); Lyster, Saito y Sato (2013) podría ser validada en términos integrales desde una perspectiva conceptual que dé cuenta de una medición cuantitativa y cualitativa del grado de conocimiento de técnicas de retroalimentación correctiva, los criterios que determinan su uso particular y la frecuencia de uso de estas instancias de intervención pedagógica.

Desde una perspectiva holística, este estudio explora las percepciones de los actores centrales en el proceso de adquisición de una segunda lengua (L2) en el contexto del programa de Pedagogía en Inglés de una universidad estatal chilena: alumnos del programa y profesores de la línea de lengua. Con el objetivo de anali- 
zar las percepciones sobre el fenómeno en estudio, se han formulado dos preguntas que guían esta investigación, a saber:

a) ¿Cuáles son las percepciones de profesores de un programa de Pedagogía en Inglés en cuanto a (i) el grado de conocimiento teórico y práctico de técnicas de retroalimentación correctiva, (ii) los criterios pedagógicos que determinan su uso y (iii) la frecuencia de uso de estas en el contexto de los cursos que conforman la línea de lengua inglesa de dicho programa?

b) ¿Cuáles son las percepciones de estudiantes de un programa de Pedagogía en Inglés en cuanto a (i) el grado de conocimiento de técnicas de retroalimentación correctiva, (ii) el reconocimiento de los criterios pedagógicos que determinan su uso y (iii) la frecuencia de uso de estas por parte de los profesores en el contexto de los cursos que conforman la línea de lengua inglesa de dicho programa?

\section{REVISIÓN DE LA LITERATURA}

El marco conceptual para este estudio no solo intenta interpretar el concepto de retroalimentación correctiva sobre la base del análisis de propuestas teóricas asociadas al concepto de error, su categorización y la medición de sus índices de frecuencia, sino que también a través de la indagación en los potenciales criterios para su uso fundados en las propuestas de interfaz de conocimiento explícito e implícito en el proceso de adquisición de una segunda lengua.

\subsection{Noción de Retroalimentación Correctiva}

Durante las últimas décadas hemos sido testigos de un innumerable aumento de estudios en la adquisición de una segunda lengua (ASL) que involucran instrucción con retroalimentación correctiva. Este constructo está directamente asociado a la noción de error cuyo origen se remonta al periodo de la psicología conductista y del audiolingüismo Corder (1981). Chaudron (1988), por su parte, la define como cualquier 'acción' por parte del profesor en respuesta a un error, que intenta informar al aprendiz sobre la presencia de este (p. 150). En una línea conceptual similar, Schachter (1991) establece que la retroalimentación correctiva, la evidencia negativa y la retroalimentación negativa son tres conceptos usados en forma indistinta en el área de adquisición de lenguas. Estas propuestas teóricas tienden a coincidir en cuanto a que la naturaleza de esta retroalimentación puede ser explícita o implícita.

Por su parte, Long (1996) ofrece una visión más comprehensiva del concepto 
de retroalimentación, enfatizando que la información que se entrega a aprendices en forma de evidencia sobre su uso de la lengua meta puede tener un carácter positivo y un carácter negativo. De esta forma, Long explica que la evidencia positiva se configura como información sobre lo que es aceptable en la estructura de la lengua meta, mientras que la evidencia negativa informa directamente a los aprendices sobre las formas que no se consideran aceptables en el uso de la lengua meta. En la misma línea, Long (2001) ofrece un marco conceptual incorporando tres tipos diferentes de evidencia negativa y positiva en relación con el ambiente lingüístico, i.e., input. De acuerdo con la clasificación anterior, la evidencia negativa y la evidencia positiva constituyen los únicos dos tipos de evidencia disponibles para el estudiante de idiomas. Cada tipo se divide adicionalmente como retroalimentación correctiva en ASL en subtipos.

La retroalimentación correctiva representa una instancia elemental en el marco de una metodología de atención a la forma. De acuerdo con Ferreira (2006), los profesores tienden a entregar retroalimentación positiva y/o negativa a sus estudiantes en los procesos de instrucción, siendo este último el más observado en estos contextos. La retroalimentación negativa o correctiva ha sido estudiada de manera extensiva, debido a la posibilidad que genera de llevar a cabo un tratamiento didáctico que permita a los estudiantes superar sus errores. Sheen (2011) entiende la retroalimentación negativa como aquella que se presenta después de una respuesta gramaticalmente incorrecta, ya sea en un espacio de instrucción formal o en un contexto natural, es decir, este tipo de retroalimentación puede ser otorgada tanto por el profesor de lenguas como por el interlocutor que posee un nivel de competencia superior que le permite notar el error.

\subsection{Estrategias de retroalimentación}

Sheen y Ellis (2011, p. 594) sugieren una taxonomía de estrategias de retroalimentación basada en la distinción de retroalimentación correctiva explícita e implícita (ver Tabla I).

Tabla I. Tipos de retroalimentación correctiva adaptada de Lyster y Saito (2010); Sheen y Ellis (2011).

\begin{tabular}{|cccc|}
\hline Clarificación Repetición & $\begin{array}{c}\text { PROMPTS } \\
\text { Señal Elicitación } \\
\text { Paralingüística }\end{array}$ & $\begin{array}{c}\text { Pista } \\
\text { Metalingüística }\end{array}$ \\
IMPLÍCITO $<$ Recast & $\begin{array}{c}\text { Recast } \\
\text { Didáctico }\end{array}$ & $\begin{array}{c}\text { Corrección } \\
\text { Explícita }\end{array}$ & $\begin{array}{c}\text { Corrección } \\
\text { Explícita (+explicación } \\
\text { Conversacional }\end{array}$ \\
& & & Metalingüística) \\
\hline
\end{tabular}


Ortega (2009) explica el concepto de explicitación desde una perspectiva que incluye las nociones de relevancia perceptual y marcación lingüística como un fenómeno variable con respecto a que las percepciones de los aprendices son determinadas, no solo por variables internas, como la edad y el conocimiento metalingüístico, sino que también por variables externas como las características propias del contexto instruccional y su eventual orientación comunicativa (Nicholas, Lightbown y Spada, 2001; Ellis y Sheen, 2006; Sato, 2011).

\subsection{Tensiones pedagógicas relacionadas con la provisión de retroalimentación correctiva}

De acuerdo con Ellis (2009), existen tensiones pedagógicas en relación con los criterios que determinan la provisión de retroalimentación correctiva determinadas por una serie de consideraciones metodológicas. Entre estas, resulta pertinente explorar aquellas relacionadas con (i) quien debe presentar esta evidencia, (ii) el grado de efectividad de los diferentes tipos de retroalimentación correctiva y (iii) el espacio temporal para la provisión efectiva de la retroalimentación correctiva.

Las caracterizaciones del proceso de provisión de retroalimentación correctiva dan cuenta de dos polos opuestos en cuanto a la fuente de retroalimentación correctiva en contextos formales. Por una parte, se enfatiza en el rol central del instructor y a la vez, el rol en los aprendices en el paradigma de algunos modelos sustentados en el enfoque en la producción de los aprendices (output) en vez de la información que estos reciben (input). En este contexto existe evidencia empírica acerca de que la generación de instancias de autocorrección facilita el proceso de adquisición de una segunda lengua L2 (Lyster, 2004; Ferris, 2006).

Otra decisión pedagógica relevante en el caso de la retroalimentación correctiva oral está relacionada con el momento en que esta evidencia debe ser provista para maximizar su efectividad. Un asunto central que determina esta decisión está directamente relacionado con el objetivo de las tareas en cuanto a estar orientadas al desarrollo de precisión en el uso de formas meta y/o el desarrollo de fluidez. Bajo este prisma, existiría una tendencia a la provisión inmediata de retroalimentación correctiva en aquellas actividades orientadas al desarrollo de precisión en el uso de una forma meta. Por otra parte, cuando las actividades están orientadas al desarrollo de fluidez, esta evidencia tiende a ser provista en forma postergada.

Los estudios recientes relacionados con las preferencias de profesores y estudiantes respecto a la provisión de retroalimentación correctiva indican que existe una relación directa entre las preferencias de los aprendices y sus conductas de aprendizaje y un grado de incongruencia entre la intención correctiva de los profesores y la interpretación de esta por parte de los aprendices, la que puede resultar en efectos negativos, en el proceso de aprendizaje de una segunda lengua. Lyster, 
Saito y Sato (2013) explican que existe una clara tendencia por parte de los aprendices en cuanto a la pertinencia de entrega de retroalimentación correctiva por parte de los instructores; no obstante, existen estudios que indican que estos grados de preferencia están directamente condicionados por el contexto cultural, las experiencias previas de aprendizaje y los niveles de competencia de los aprendices.

De acuerdo con Jean y Simard (2011), los profesores expresan una preferencia por la entrega de retroalimentación correctiva solo cuando los errores impiden la comunicación para no interferir con la comunicación y no afectar los niveles de confianza de los aprendices De este modo, la provisión de la retroalimentación correctiva parece estar determinada por dos factores: (i) el efecto negativo en la naturaleza comunicativa de algunas tareas Brown (2009) y (ii) la relación de esta con los niveles de ansiedad causados por el hecho de ser corregidos en público Lasagabaster y Sierra (2005).

\subsection{Interfaz implícita y explícita en el proceso de adquisición de una segunda lengua}

Una caracterización pertinente del repertorio de técnicas de retroalimentación correctiva junto con los criterios pedagógicos que determinan su uso y la frecuencia debe entender el fenómeno de retroalimentación correctiva como un proceso de intervención pedagógica implícita y/o explícita, como una respuesta sistemática a enunciados que contienen una o más formas erróneas producidos por aprendices de una segunda lengua (L2). En este contexto, el grado de explicitación de la retroalimentación correctiva parece constituir el sustento teórico que informa las decisiones pedagógicas de los profesores de lengua en cuanto a: (i) el tipo de retroalimentación correctiva y su frecuencia de uso, (ii) la optimización de la evidencia presentada a través de la retroalimentación correctiva para optimizar las condiciones que faciliten los procesos cognitivos de atención a esta y a la interpretación de su intención correctiva por parte de los aprendices y (iii) un factor relevante para la medición empírica del efecto de la retroalimentación correctiva en términos del grado de asimilación de la evidencia presentada por parte de los aprendices.

En este sentido Ellis (2002) plantea la necesidad de indagar en la naturaleza de las representaciones lingüísticas implícitas y la forma en que estas resultan en el uso fluido y preciso de una segunda lengua (L2). Asimismo, se considera pertinente explorar el proceso de configuración de estas representaciones y cómo el procesamiento de formas lingüísticas emerge del uso de estas. En las bases teóricas recientes existe un amplio consenso acerca de que una vasta parte del procesamiento lingüístico es inconsciente y que los procesos cognitivos están determinados por el producto de procesos de aprendizaje implícito (Ellis, 2011). Durante este proceso, la frecuencia de uso de una forma determina la disponibilidad de acceso a 
las representaciones lingüísticas. Durante el proceso de aprendizaje implícito se registra evidencia lingüística que permite resolver el problema de asociación entre una forma y su función en contexto.

\subsection{Rol de la retroalimentación correctiva}

En relación con las acciones de intervención pedagógica de carácter explícito orientadas a superar las rutinas implícitas que no son óptimas para el proceso de adquisición de la L2, el rol de la retroalimentación correctiva es central. En este contexto, se hace necesario indagar si las representaciones implícitas y explícitas constituyen efectivamente sistemas distintos, o si existen etapas intermedias en la representación lingüística, y si las representaciones implícitas pueden convertirse en explícitas y viceversa.

En este escenario, el rango de interpretaciones varía desde la postura categórica de Krashen (1982), Paradis (2004) y Ellis (2009), quienes caracterizan las representaciones implícitas y explícitas como sistemas independientes y autónomos; Karmiloff- Smith (1995) y Dienes y Perner (1999) describen estas representaciones lingüísticas en un continuo.

(1) La posición de no-interfaz plantea que la transferencia entre conocimiento explícito e implícito no es posible en cuanto existen diferencias en la forma en que estas son adquiridas (Krashen, 1982); almacenadas (Paradis, 1994, 2004) y activadas (Ellis, 1993).

(2) La posición de interfaz fuerte plantea que la reconfiguración de representaciones explícitas en representaciones implícitas y viceversa, está condicionada por procesos de automatización a través de práctica (DeKeyser, 1998, 2003, 2007).

(3) La posición de interfaz débil acepta el planteamiento que la reconfiguración de representaciones implícitas en explícitas y viceversa; sin embargo, esta postura asume que este proceso de reconfiguración está condicionado por la presencia de prerrequisitos psicolingüísticos para la adquisición de una forma en una etapa determinada de desarrollo intralingüístico (Pienemann, 1989). Las representaciones explícitas contribuyen a la internalización de representaciones implícitas a través de la activación de procesos cognitivos de 'noticing' y analogías Ellis (2006), o facilita la producción, la que a su vez constituye input para los procesos cognitivos implícitos generando representaciones implícitas Schmidt y Frota (1986).

En forma complementaria a los marcos conceptuales y taxonomías relacionadas con el objeto de este estudio, algunos estudios han explorado variables al- 
ternativas que dan cuenta de los grados de efectividad del proceso correctivo. En este sentido, Sato y Loewen (2018) exploran el impacto de la retroalimentación correctiva en el contexto de la instrucción metacognitiva con un enfoque en uso de recast conversacional y clarificaciones (clarification requests) en el contexto del proceso de adquisición de la inflexión para la tercera persona en inglés y los posesivos 'his' y 'her'. Los autores concluyen que los grados de efectividad de la retroalimentación correctiva dependen, en este ámbito instruccional, de la naturaleza de la estructura meta.

Una aproximación alternativa explora y caracteriza el proceso de retroalimentación correctiva presentada con tareas de interacción entre aprendices de una segunda lengua (L2), particularmente en términos del efecto la instrucción formal con respecto a los criterios que determinan la provisión de la retroalimentación correctiva. En este contexto, Fujii, Ziegler y Mackey (2016) sugieren la implementación de una secuencia de etapas para el desarrollo de grados de autonomía por parte de los aprendices de una segunda lengua (L2) para el procesamiento de la información correctiva. Dicha secuencia, implementada en el contexto instruccional de aprendices japoneses de inglés como segunda lengua, se estructura en base a la presentación explícita de los fundamentos del enfoque comunicativo (CLT) en conjunto con información explícita de patrones de interacción y métodos de provisión de retroalimentación correctiva. Los resultados obtenidos en forma posterior a la participación activa de los aprendices en el proceso de provisión de retroalimentación correctiva revelan que la frecuencia de provisión de esta se incrementa en forma significativa, sugiriendo que un conocimiento mayor por parte de los aprendices de la naturaleza de la retroalimentación correctiva resulta en un nivel de interacción cualitativamente superior para el desarrollo de una segunda lengua. Estos resultados son coherentes con los presentados por Sato y Lyster (2012) en su estudio exploratorio del efecto de un periodo de 10 semanas de instrucción sobre la naturaleza de la retroalimentación correctiva a aprendices de una segunda lengua. Este estudio se estructura en base a la participación de los aprendices en discusiones guiadas sobre sus percepciones acerca del rol de la retroalimentación correctiva en el proceso de adquisición de una segunda lengua, seguido de un proceso de ejemplificación de las diferentes estrategias de retroalimentación correctiva complementado con sesiones prácticas.

\section{METODOLOGÍA}

Este estudio de caso presenta un enfoque cuali-cuantitativo no experimental, desde un paradigma interpretativo, pretende explorar y describir las percepciones de los alumnos y profesores de una carrera de Pedagogía en Inglés con relación a la retroalimentación oral correctiva que se utiliza en las cátedras de lengua inglesa. 


\subsection{Participantes}

La muestra estuvo constituida por los alumnos y profesores de dicho programa. Los alumnos provienen de contextos socioeconómicos variados y con un nivel de competencia lingüístico heterogéneo. El objetivo del programa es que los alumnos desarrollen competencias a nivel C1 del Marco Común Europeo de Referencia para las lenguas (MCER). En cuanto a los docentes, todos corresponden a profesionales con el título de profesor de inglés y licenciados en lengua inglesa. Algunos poseen estudios de postgrado y vasta experiencia, mientras otros son licenciados con poca experiencia docente.

\subsection{Universo y muestra}

Este estudio consideró la población constituida por la totalidad de estudiantes de los niveles $1^{\circ}, 2^{\circ}, 3^{\circ}$ y $4^{\circ}$ años de la Pedagogía en Inglés, y una muestra representativa no probabilística de profesores de dicha carrera. El universo posible era de 188 alumnos y 10 profesores. La muestra corresponde a 149 estudiantes y a 10 profesores del programa. Solo participaron los alumnos de $2^{\circ}$ a $5^{\circ}$ año. Por su parte, los profesores debían estar impartiendo clases de lengua al momento de la aplicación de los instrumentos de recolección de datos y pertenecer al grupo de profesores por hora y jornada asociados al programa. En este caso, hubo un total de 6 participantes, 2 hombres y 4 mujeres, de un universo posible de 10 profesores.

\subsection{Instrumentos de recolección de datos}

Se aplicaron dos instrumentos a lo largo del estudio. El primero consistió en una encuesta anónima aplicada directamente a los alumnos y profesores para identificar las percepciones y experiencias sobre la retroalimentación oral correctiva. La matriz sobre la cual se estructuró la encuesta se observa en la Tabla II.

Tabla II. Matriz de elaboración de cuestionarios.

\begin{tabular}{|l|l|l|l|}
\hline Dimensión & Subdimensiones & Descriptores & Tipo reactivo \\
\hline $\begin{array}{l}\text { Tipo de corrección } \\
\text { en discurso oral }\end{array}$ & Tipo & $\begin{array}{l}\text { Identificar tipo } \\
\text { de correctivos }\end{array}$ & Selección múltiple \\
\cline { 2 - 4 } & Frecuencia & $\begin{array}{l}\text { Identificar } \\
\text { frecuencia }\end{array}$ & Escala Likert \\
\cline { 3 - 4 } & $\begin{array}{l}\text { Identificar } \\
\text { presencia }\end{array}$ & $\begin{array}{l}\text { Preguntas } \\
\text { dicotómicas }\end{array}$ \\
\hline
\end{tabular}


Posteriormente a la entrevista, se organizaron tres focus groups con el fin de expandir las representaciones exploradas en la encuesta, así como para poder corroborar la información recolectada en la etapa previa. Los focus groups se realizaron en sesiones de 40 minutos con una entrevista semiestructurada para garantizar la presencia de elementos clave.

\subsection{Procesamiento de la información}

Los datos de las encuestas fueron tabulados en una planilla Excel y se analizó la frecuencia de las respuestas utilizando el software estadístico SPSS . Las entrevistas de los focus groups fueron grabadas en dispositivos digitales y luego transcritas y su contenido analizado colectivamente por el equipo de investigación, mediante el levantamiento de categorías cualitativas consensuadas, en reuniones para dicho efecto.

\subsection{Lineamientos éticos}

Todos los participantes firmaron un consentimiento informado al inicio del estudio donde se garantizó su anonimato y confidencialidad de las opiniones vertidas tanto en la encuesta como en el focus group. Todos los procedimientos se llevaron a cabo según los lineamientos establecidos por la Comisión de Ética de la Universidad de Santiago de Chile.

\section{ANÁLISIS Y DISCUSIÓN DE LOS RESULTADOS}

Con el propósito de facilitar la lectura y comprensión de los resultados obtenidos de las encuestas y los focus groups a profesores y estudiantes, se ha optado por presentar estos resultados en dos secciones diferentes: (4.1.) resultados de las encuestas escritas y (4.2.) resultados de las entrevistas realizadas en los grupos focales.

\subsection{Resultados de las encuestas escritas}

En lo que sigue se presentarán los resultados obtenidos desde la aplicación de las encuestas escritas. Estas se presentarán señalando lo que dicen los profesores primero para contrastarlo después con la opinión de los estudiantes. 
Tabla III. Recepción o entrega de retroalimentación por parte de alumnos y profesores.

\begin{tabular}{|l|l|l|}
\hline \multicolumn{2}{|l|}{ Reciben o entregan retroalimentación } \\
\hline Respuestas & Sí & No \\
\hline Estudiantes & $86 \%$ & $14 \%$ \\
\hline Profesores & $67 \%$ & $33 \%$ \\
\hline
\end{tabular}

Los resultados en la Tabla III indican que tanto profesores como estudiantes coinciden en que se entrega o recibe retroalimentación durante las clases de inglés, 67 y $86 \%$, respectivamente.

Tabla IV. Frecuencia con la que se realiza la retroalimentación por agente.

\begin{tabular}{|l|l|l|l|l|l|}
\hline Frecuencia & Esporádicamente & $\begin{array}{l}\text { Algunas } \\
\text { veces }\end{array}$ & Frecuentemente & $\begin{array}{l}\text { Casi } \\
\text { siempre }\end{array}$ & Siempre \\
\hline Estudiantes & $3,4 \%$ & $25,3 \%$ & $37 \%$ & $24,6 \%$ & $9,6 \%$ \\
\hline Profesores & $0 \%$ & $0 \%$ & $17 \%$ & $67 \%$ & $33 \%$ \\
\hline
\end{tabular}

En relación con la frecuencia con la que los profesores entregan retroalimentación y la reciben los estudiantes, los resultados muestran que el $67 \%$ de los profesores reconocen entregarla casi siempre y un 33\% dice entregarla siempre. En tanto, los estudiantes reconocen recibirla frecuentemente (37\%) algunas veces $(25,3 \%)$ y casi siempre $(24,6 \%)$.

Tabla V. Tipo de retroalimentación que se entrega o recibe.

\section{Tipo de retroalimentación}

\begin{tabular}{|l|l|l|l|}
\hline Respuesta & Explícita & Implícita & Ambos \\
\hline Estudiantes & $18,7 \%$ & $20,3 \%$ & $60,9 \%$ \\
\hline Profesores & $17 \%$ & $0 \%$ & $83 \%$ \\
\hline
\end{tabular}

La Tabla $\mathrm{V}$ muestra que tanto los estudiantes y profesores coinciden en que, durante el aprendizaje del idioma inglés, los profesores proveen retroalimentación de tipo explícita e implícita. Esto es refrendado en un $83 \%$ por los profesores y un $60,9 \%$ por los estudiantes. 
Tabla VI. Momento de la retroalimentación.

\begin{tabular}{|l|l|l|}
\hline \multicolumn{3}{|l|}{ Cuando usted recibe retroalimentación esta es... } \\
\hline Respuesta & Inmediata & No inmediata \\
\hline Estudiantes & $80,5 \%$ & $19,5 \%$ \\
\hline Profesores & $83 \%$ & $17 \%$ \\
\hline
\end{tabular}

La Tabla VI presenta el momento en que se lleva a cabo la retroalimentación. Un $83 \%$ de los profesores señala que se realiza de manera inmediata y solo un $17 \%$ indica que se lleva a cabo off-line. Por su parte, los estudiantes coinciden con estos resultados, señalando que un $80,5 \%$ recibe retroalimentación oral correctiva inmediata, mientras que solo un $19,5 \%$ dice que la recibe en otro momento.

Tabla VII. Tipos de correctivos recibidos o entregados.

\begin{tabular}{|l|l|l|l|l|l|}
\hline Tipo de retroalimentación que usted recibe o entrega \\
\hline Respuestas & $\begin{array}{l}\text { Reformulación } \\
\text { correctiva }\end{array}$ & Repetición & $\begin{array}{l}\text { Pistas } \\
\text { metalingüísticas }\end{array}$ & Clarificación & Omisión \\
\hline Estudiantes & $81,2 \%$ & $83,2 \%$ & $42,2 \%$ & $79,8 \%$ & $6,7 \%$ \\
\hline Profesores & $100 \%$ & $83 \%$ & $50 \%$ & $100 \%$ & $0 \%$ \\
\hline
\end{tabular}

La Tabla VII muestra que el 100\% de los profesores admiten que utilizan la reformulación correctiva y la clarificación, lo que es refrendado por los estudiantes con un 81,2 y $79 \%$, respectivamente. A estos dos tipos, le sigue la repetición y la entrega de pistas metalingüísticas. En lo que sigue se presentan los resultados obtenidos en las entrevistas de los grupos focales.

\subsection{Resultado de los grupos focales}

Para propósitos del análisis, se seleccionó de entre las preguntas cuyas respuestas responden directamente a las dos interrogantes de este estudio, en concreto 0,6 , 7, 8, 9, 10 y 11 desde la mirada de los profesores y los alumnos. Sin perjuicio de lo anterior, los datos de las otras preguntas se encuentran disponibles en caso de ser solicitados por la comunidad investigadora.

Los resultados de los tres grupos focales se presentan agrupados por categorías y ordenados según la frecuencia con que fueron enunciados por los profesores y 
estudiantes participantes. Además, con el propósito de ilustrar las categorías establecidas y sustentar los argumentos entregados por los participantes, se transcriben extractos de aquellas intervenciones que se consideró representan mejor la categoría, o constituyen un ejemplo significativo a la pregunta hecha.

Pregunta 0 (Solo Estudiantes): ¿Qué estrategias de retroalimentación correctiva consideran ustedes son las más efectivas?

Tabla VIII. Pregunta 0. Tipo de retroalimentación más efectiva.

\begin{tabular}{|l|l|}
\hline Categorías & Frecuencia \\
\hline Pistas metalingüísticas & 4 \\
\hline Repetición & 3 \\
\hline Corrección inmediata & 2 \\
\hline Afectivas & 2 \\
\hline
\end{tabular}

Como se observa en la Tabla VIII, los estudiantes expresan que las estrategias más efectivas son aquellas en las que el profesor, de manera explícita o implícita, intenta que el alumno se percate que ha cometido un error en su participación, entregando algunas pistas metalingüísticas. "El profesor marcaba el error, entonces el alumno se daba cuenta y entonces cambiaba", "la que más recuerdo de los profes era el uso de la mano para señalar las estructuras gramaticales en caso de que uno llegase a omitir una”.

Otra estrategia mencionada corresponde a la solicitud de repetición del enunciado que contiene un error, por parte del profesor, con el propósito explícito de que el estudiante corrija el error. También se mencionan como estrategias efectivas aquellas que se hacen de manera inmediata, una vez cometido el error, sin llegar a identificar o precisar si esta se debiera hacer al momento exacto de cometer el error, interrumpiendo al estudiante, o si se hace una vez terminada la participación del estudiante. Así, señalan que "las más efectivas son las que se hacen de inmediato, cuando se comete el error".

Otra respuesta obtenida dice relación con la forma en que la retroalimentación se debiera entregar para no generar un sentimiento de culpabilidad o vergüenza que inhiba la participación posterior del estudiante en la clase. A esta condición la hemos llamado afectividad de la retroalimentación, tal como se evidencia en el discurso de los estudiantes: "creo que las más efectivas son las afectivas porque, una cosa, ya, es golpear (la mesa), pero si un profesor golpea así de repente y asusta a unos alumnos. Es mejor hacerlos (sic) con tino". En efecto, la pertinencia de la empatía con el alumno es un elemento saliente: "claro, con tacto, eso para mí es 
lo más afectivo y que puede ser cualquiera de las técnicas, pero siempre teniendo en cuenta... lo afectivo".

Pregunta 6: (Estudiantes) ¿Los profesores les informan a ustedes del tipo de retroalimentación que usan en clases?

Tabla IX. Tipo de retroalimentación utilizada.

\begin{tabular}{|l|l|l|}
\hline $\begin{array}{l}\text { (Profesores) ¿Ustedes informan a los estudiantes } \\
\text { sobre el tipo de retroalimentación que usan en } \\
\text { clases? Categorías }\end{array}$ & Estudiantes & Profesores \\
\hline Se usa retroalimentación, pero no se explicita & 5 & 1 \\
\hline No & 2 & 4 \\
\hline
\end{tabular}

Los datos presentados en la Tabla IX revelan que los profesores, en su mayoría, proveen retroalimentación a los estudiantes, pero no explicitan o hacen notar a los estudiantes que, durante las interacciones orales, ellos/as utilizarán diferentes estrategias para corregir, implícita o explícitamente los errores cometidos por los estudiantes: "La retroalimentación existe en cada clase..., pero jamás se nos dice, así como "mi retroalimentación es tal". "Como que nunca se presenta la retroalimentación que se va a hacer". En este sentido, enfatizan la pertinencia de una inducción a los modos de corrección al inicio del curso: “....al principio del semestre, la profesora debiera informarlo para que los estudiantes sepamos reconocer cuándo se nos están corrigiendo, cuándo están de acuerdo con nosotros y qué tipo de error", "debieran enseñarnos en el primer semestre, cuáles son las posibles formas de corrección, porque así uno puede ir evitando que ese error se haga hábito..., [y] que tengamos un margen o ... una lista, por decirlo si, de formas de corrección”. Esta falta de concientización por parte del alumnado genera que, en algunos casos, los estudiantes expresen que los profesores no utilizan estrategias de retroalimentación: "muchas veces pasa desapercibida la retroalimentación", "yo no recuerdo ninguna instancia donde me hayan explicado anteriormente cómo iba a ser la corrección”. Otras respuestas complementan la problemática, en cuanto a que asocian la frecuencia de retroalimentación a la didáctica empleada por cada profesor y, en este sentido, cuando la presentación de la retroalimentación se presenta esporádicamente parece provocar confusión en el alumnado: "Los profesores deberían ponerte un margen para que no se confunda...", "uno no sabe a veces si el profesor está de acuerdo contigo o te está corrigiendo..."

En la misma línea de análisis, las profesoras declaran principalmente que 1) entregan retroalimentación a los estudiantes como estrategia en el proceso de en- 
señanza y 2) reconocen que no informan a los estudiantes cuando y qué tipo de retroalimentación se entrega a los estudiantes. En relación con este último aspecto, algunos profesores asumen que no sería necesario informar a estudiantes, ya que, a través de la acción correctiva, el estudiante se debiese dar cuenta de su error de producción. De este modo, "ellos [sabrían] cómo [corregirse] en un ambiente más natural y [arreglar] lo que ellos dicen”. Más aún, enfatizan que dicho proceso metacognitivo no debiese ser informado por el docente. De esta forma, reflexiones en cuanto a este punto incluyen: “¿Por qué les tengo que decir sí voy a corregir?" "Si... es de manera implícita, no le informo, no le advierto...". No obstante, señalan que la corrección sí es de manera explícita en cursos de redacción o se encuentra presente en los criterios de evaluación de las rúbricas.

En el caso de los profesores que afirman retroalimentar argumentan que realizan retroalimentación de manera formativa, pero que no realizan ningún tipo de inducción al alumno, lo que produce que se cuestionen hasta qué punto los alumnos de cursos inferiores realmente están al tanto de la retroalimentación entregada por los docentes. Es interesante señalar que, durante la entrevista, se generó una instancia de reflexión colaborativa que tuvo por resultado un posible cambio en la cognición docente en relación con este punto. De esta manera, algunos profesores que no retroalimentaban por desconocimiento de estrategias rescataron la utilidad que podría tener una sistematización de dicho proceso en clases: "quizás sería una buena opción decirles como 'yo corrijo de esta forma'...nunca se me había ocurrido [una inducción]... para que no se sientan como atacados". Lo anterior se condice con la condición de afectividad en la retroalimentación identificada previamente por los alumnos, por lo que se perfila como un elemento importante para maximizar el efecto de la corrección en el aula de L2.

Pregunta 7: (Estudiantes) ¿Los profesores evalúan el efecto de retroalimentación correctiva que entregan en clases?

(Profesores) ¿ Ustedes evalúan el efecto de la retroalimentación correctiva que entregan en clases?

Tabla X. Evaluación del efecto de la retroalimentación.

\begin{tabular}{|l|l|l|}
\hline \multirow{2}{*}{ Categorías } & Frecuencia \\
\cline { 2 - 3 } & Estudiantes & Profesores \\
\hline Sí & 2 & 5 \\
\hline No & 2 & 0 \\
\hline
\end{tabular}

Las respuestas a esta pregunta permiten establecer que la retroalimentación 
correctiva oral en el aula constituye de por sí una subcategoría en que los alumnos clasifican a sus profesores, vale decir, aquellos que efectivamente lo hacen y aquellos que no (ver Tabla X). No obstante, advierten que, a pesar de ser identificado y corregido, no existe seguimiento efectivo durante las clases que garantice la eliminación de dicho error del discurso del estudiante: "hay profesores que corrigen en el momento y después se olvidan de que tuviste ese error y te lo corrigen de la misma forma o con distinta estrategia, pero, aun así, después no se cercioran”.

Los estudiantes, además, hacen la diferenciación entre la retroalimentación correctiva y una retroalimentación "afectivo-positiva" (acuñada por ellos), donde señalan que es igual de importante que el profesor felicite o valore el correcto uso de la lengua o los aportes de los estudiantes en las clases. "Creo que también es súper importante que nos den feedback cuando hacemos cosas buenas, porque yo, sinceramente, he visto presentaciones de compañeros durante estos años que han sido de verdad muy buenas y los profes les han dicho como 'sí, buena información, thanks'. Entonces, ¿̇buena información?”. En consecuencia, la incorporación de estrategias de retroalimentación afectivo-positiva podría contribuir a un mejor clima de aula y configuración de seguridad en el proceso de aprendizaje de la lengua extranjera.

Las respuestas de los estudiantes se contradicen con las de las profesoras participantes, quienes unánimemente coinciden en que sí existe una evaluación del efecto de la retroalimentación que entregan a sus estudiantes. En este sentido, establecen que existen oportunidades posteriores a la retroalimentación para que este vuelva a producir de manera correcta en un contexto dado y, de esta manera, evaluar si efectivamente el error fue corregido por el estudiante. Del mismo modo, señalan que, si la retroalimentación implícita parece no funcionar, esta adquiere carácter de explícita y pasa a ser compartida con el resto de la clase. No obstante, no establecen los criterios mediante los cuales se determina cuándo un error debiese pasar de modalidad implícita a explícita.

Pregunta 8 (Estudiantes): ¿Cómo reciben ustedes la retroalimentación correctiva entregada por sus profesores? ¿En forma positiva o negativa?

Tabla XI. Evaluación de la retroalimentación entregada.

\begin{tabular}{|l|l|}
\hline Categorías & Frecuencia \\
\hline Positivamente & 5 \\
\hline Negativamente & 5 \\
\hline
\end{tabular}

$\mathrm{Al}$ consultar a los estudiantes sobre las percepciones que provocan en ellos las 
correcciones explícitas o implícitas a sus errores cometidos en las interacciones, se observan percepciones porcentualmente igualitarias. Para algunos estudiantes, la retroalimentación se asume positivamente: “...aprendí a valorar mucho más la corrección, incluso la buscaba, en fonética me acuerdo de que de repente me molestaba cuando no la hacían...". "Los profesores lo hacían con el afán de que uno aprendiera y después si no cometía el error, te felicitaban". "Tenía una profesora de fonética que era dulce, totalmente dulce, nos hacía leer y cuando yo me equivocaba, ella me decía "otra vez, otra vez", hasta que yo lo podía hacer. Y era la forma más dulce en que alguien podía tratar a una persona tímida como yo" (ver Tabla XI).

No obstante, otros estudiantes reciben la retroalimentación de forma negativa. Dicha subjetividad tiene efectos directos en 1) la experiencia del aula ("Era como que yo estaba haciendo todo mal, y se iba todo pa' abajo y la clase ya era... se convertía en una tortuosa experiencia”); 2) relación afectiva con el profesor (“depende muchísimo de cómo te está retroalimentando... la profesora era tan desagradable, antipática que a uno le daba miedo cometer error"); y 3) percepción afectiva de la retroalimentación ("Hay profesores que son muy duros en la forma de corregir que a uno lo marcan y aprende del error, pero te marcan para mal"). En este sentido, enfatizan que debe haber una correspondencia entre la retroalimentación entregada por el profesor y el resultado en evaluaciones sumativas.

Pregunta 8 (Profesores): ¿Qué tan necesarios son el conocimiento y dominio de estrategias de retroalimentación para un profesor?

Tabla XII. Evaluación de la importancia del conocimiento y dominio de estrategias de retroalimentación para un profesor.

\begin{tabular}{|l|l|}
\hline Categorías & Frecuencia \\
\hline Muy relevante & 5 \\
\hline
\end{tabular}

Por su parte, todas las profesoras participantes coinciden en que el conocimiento y dominio de las estrategias de retroalimentación son relevantes porque facilitan el proceso de enseñanza, y el aprendizaje de los estudiantes "...porque de eso depende de que en una clase cada alumno haya corregido ciertos errores o, quizás, la estructura que se ve en clases... Son súper importantes, ya que de eso depende el avance del alumno, conocer técnicas que no sean punitivas tampoco...”. En relación con este punto, agregan que el conocimiento de un repertorio diverso de formas de retroalimentación permite adaptarse a las características de los alumnos (ver Tabla XII). 
Las respuestas también evidencian la carga asociada a las representaciones de la retroalimentación explícita e implícita. La primera está asociada a una condición más bien punitiva para el estudiante, en cuanto a que se evidencia públicamente la presencia de un error en el discurso de un estudiante, mientras que el segundo tipo goza de una aparente idealización, en cuanto a que promueve la continuidad discursiva de los estudiantes, así como también el desarrollo de estrategias metacognitivas para la evaluación de su propia producción. Lo anterior está condicionado a la cantidad de estrategias a utilizar, así como del momento en que se realiza la retroalimentación. Es importante destacar que algunas de las respuestas docentes apuntan a que la percepción de negatividad o positividad de la retroalimentación está ligada al tipo realizado por el docente, vale decir, explícito o implícito, respectivamente. No obstante, esto no se condice con los alumnos, quienes dentro de la retroalimentación implícita tipifican las diferentes reacciones hacia la corrección.

Pregunta 9 (Profesores): ¿Qué tipo de retroalimentación consideran ustedes son las más efectivas?

Tabla XIII. Efectividad de los tipos de retroalimentación.

\begin{tabular}{|l|l|}
\hline Categorías & Frecuencia \\
\hline Parafraseo & 3 \\
\hline Corrección explícita & 1 \\
\hline Todas & 1 \\
\hline
\end{tabular}

Las profesoras afirman que durante sus clases utilizan diferentes estrategias de retroalimentación, enunciando de manera indirecta algunas de las estrategias propuestas por Lyster y Ranta (1997). Para algunas profesoras, el parafraseo es la estrategia más utilizada, incorporando en ellas el uso de la pizarra para analizar el error. Todas convergen en que primero parafrasean explícitamente la estructura en cuestión para luego escribirla en la pizarra con el fin de promover el desarrollo metacognitivo de los estudiantes. Esto posteriormente es seguido por práctica oral de pronunciación de ser necesario. En sus propios relatos, describen "el parafraseo... como [el] top one, el que más frecuente utilizo. Y, en forma explícita, ya llevando el ejemplo directo a la pizarra, preguntando a los chicos si notan algún ejemplo o algún error que podemos mejorar de eso o practicando pronunciación si es que fuese el caso". Otra profesora, de manera muy similar, señaló que "en mi caso, también parafraseando, haciendo que ellos se corrijan entre ellos, así como en grupo, ese tipo de cosas y, luego... llevando el error directamente a la pizarra o que yo repito lo que ellos dicen". En este último caso, el proceso de retroalimenta- 
ción comprendería una etapa previa a la explicitación en que se da la oportunidad de corrección entre pares para promover el aprendizaje y negociación colectiva del contenido y del error (ver Tabla XIII).

Otro uso del error con propósito correctivo corresponde a tomar notas de los errores cometidos por los estudiantes y concluir la clase realizando una retroalimentación abierta a todo el curso. “... yo dejo los errores que (sic) sigan cuando hay trabajos en grupo, y después al final yo les corrijo todo y lo escribo en la pizarra y digo como "final activities for the mistake" y entre todos identifican y dicen "ay, eso lo dije yo" o, no sé, después se llevan como el listado correcto y, a la otra clase, empezamos con temas así para que usen las mismas estructuras y así se recuerde el error". Lo anterior evidencia una conciencia de la pertinencia de la corrección según el propósito de la actividad, así como de la importancia de la metacognición en el centro de la reflexión durante el proceso de retroalimentación.

Pregunta 10 (Profesores) ¿Cómo han adquirido o adquirieron ustedes sus conocimientos sobre la retroalimentación correctiva?

Tabla XIV. Origen del conocimiento sobre retroalimentación correctiva.

\begin{tabular}{|l|l|}
\hline Categorías & Frecuencia \\
\hline Formación inicial & 5 \\
\hline En la práctica docente & 5 \\
\hline
\end{tabular}

Respecto a esta pregunta, las profesoras admiten que mantienen interés permanente por informarse o estar actualizadas en este tema. Todas reconocen haber recibido información durante su formación profesional en pregrado, haber probado diferentes estrategias durante su desarrollo profesional y haber continuado informándose o perfeccionando sus habilidades para proveer retroalimentación a sus estudiantes. "[...] en el curso primero de Metodología, luego a través de la experiencia, poniendo en práctica esto y dándome cuenta cuál sirve, cuál está mejor orientada al tipo de clase, al tipo de alumno. En la práctica, propiamente tal". "A través de los estudios acá, de haber estudiado eso en Metodología, de lectura personal y a través del recuerdo que tengo yo de algunos profesores también". "[...] en el curso de Metodología en pregrado y después en la práctica”. De esta forma, se puede concluir que el conocimiento pedagógico sobre la retroalimentación obedece a varios factores que enriquecen el repertorio de estrategias, pertinencia y significatividad de la misma. En consecuencia, resulta complejo poder establecer una única razón para establecer el uso de una estrategia de retroalimentación. 
Pregunta 11 (Profesores) Considerando que viene esto de pregrado, que ya pasó un tiempo, ¿cómo actualizan ustedes su repertorio de técnicas de retroalimentación correctiva?

Tabla XV. Percepción acerca de la actualización del repertorio de técnicas de retroalimentación correctiva.

\begin{tabular}{|l|l|}
\hline Categorías & Frecuencia \\
\hline Asistiendo a seminarios, capacitaciones, charlas, & 4 \\
\hline A través de lecturas en la Internet & 2 \\
\hline Lecturas personales & 1 \\
\hline Cursos on line & 1 \\
\hline
\end{tabular}

Como se observa en la Tabla XV, diferentes han sido las acciones realizadas por las profesoras para actualizar su repertorio de estrategias de retroalimentación. En este sentido, destacan: 1) la participación en instancias de discusión académica ("...asistiendo a congresos o charlas o capacitaciones, y lecturas por curiosidad...”), 2) capacitación online (“...[realizando] cursos online que son específicos con temática de corrección o giving feedback...”), 3) lectura académica online (“... también leyendo en la Internet, actualizando contenido”), y 4) el aprendizaje en aula (“...en la práctica, dándome cuenta de que a veces esa corrección no era la adecuada y que debo mejorarla...”). La combinación de estos elementos promueve el desarrollo de estrategias propias para la corrección y retroalimentación como resultado de una práctica reflexiva.

\section{CONCLUSIONES}

Este estudio examinó las percepciones de profesores y estudiantes de un programa de Pedagogía en Inglés acerca de la retroalimentación oral correctiva en las clases de lengua inglesa. Las preguntas de investigación que guiaron el estudio fueron las siguientes:

a) ¿Cuáles son las percepciones de los profesores de un programa de Pedagogía en Inglés en cuanto a (i) el grado de conocimiento teórico y práctico de técnicas de retroalimentación correctiva, (ii) los criterios pedagógicos que determinan su uso y (iii) la frecuencia de uso de estas en el contexto de los cursos que conforman la línea de lengua inglesa de dicho programa? 
b) ¿Cuáles son las percepciones de los estudiantes de un programa de Pedagogía en Inglés en cuanto a (i) el grado de conocimiento de técnicas de retroalimentación correctiva, (ii) el reconocimiento de los criterios pedagógicos que determinan su uso y (iii) la frecuencia de uso de estas por parte de los profesores en el contexto de los cursos que conforman la línea de lengua inglesa de dicho programa?

En cuanto a la primera pregunta, los resultados obtenidos desde las entrevistas, administradas mediante focus groups, evidencian que los profesores poseen un alto grado de conocimiento teórico y práctico de técnicas de retroalimentación correctiva. Sin embargo, no existen criterios pedagógicos precisos que determinan su uso, y este está determinado por elementos relacionados con la naturaleza de las clases.

Con respecto a la frecuencia de uso de la corrección oral correctiva en el contexto de los cursos que conforman la línea de lengua inglesa, un $67 \%$ de los profesores la utilizan en aula, lo que permite concluir que existe una práctica instalada de proveer retroalimentación correctiva en clases. Asimismo, los resultados señalan que el $100 \%$ de los profesores entrega la retroalimentación oral correctiva de manera inmediata y solo un $19,5 \%$ lo hace de manera diferida.

En cuanto a la segunda pregunta, los estudiantes indican desconocer las estrategias de retroalimentación correctiva utilizadas por los profesores y los criterios pedagógicos para su uso. Sin embargo, los estudiantes perciben en un $86 \%$ el uso de la retroalimentación correctiva por parte de sus profesores en el aula. Con respecto al tipo de retroalimentación utilizada, los estudiantes en un $80,5 \%$ están de acuerdo en que la reciben de manera inmediata en clases y solo un $14 \%$ la recibe offline. En relación con el tipo de estrategia de retroalimentación oral correctiva recibida, los estudiantes señalan que identifican en la clase de lengua, un alto uso de la "retroalimentación correctiva explícita", la "repetición, "la clarificación", las "pistas metalingüísticas" y la "omisión".

Un hallazgo significativo dice relación con la percepción de la importancia de la entrega de retroalimentación correctiva, por parte de los profesores en este programa de Pedagogía en Inglés. Ellos ponen énfasis en la necesidad de que este sea explícito en naturaleza, a pesar de ser usado en el área de lengua, o que presupone un grado de atención a significado por sobre dimensiones formales explícitas que favorecen la atención a la forma. Por su parte, los estudiantes reconocen que probablemente el bajo nivel lingüístico de algunos alumnos les impediría reconocer la intención correctiva de la información entregada por los profesores, afectando, con esto, el grado de atención a la misma, en un contexto de instrucción formal, y el potencial nivel de asimilación de la evidencia presentada a través de la instancia de retroalimentación correctiva, uptake.

Por otra parte, los resultados de este estudio indicarían que existiría una falta 
de sistematización en el procedimiento de entrega de retroalimentación correctiva oral, en las clases de lengua inglesa. Esta situación generaría altos niveles de ansiedad en los estudiantes durante el proceso de aprendizaje, lo que resultaría en la incorrecta interpretación de las instancias de retroalimentación correctiva, generando incluso niveles más altos de ansiedad por parte de los alumnos.

Considerando todas las ideas planteadas anteriormente, se concluye que es necesario generar instancias de inducción formal para los estudiantes del programa de Pedagogía en Inglés, del repertorio de estrategias de retroalimentación oral correctiva usadas, por los profesores, en el contexto del área de lengua de dicha carrera. Esta instancia permitiría establecer criterios pedagógicos estandarizados y sistemáticos para el uso de las estrategias de retroalimentación oral correctiva, conocimiento que debiera ser compartido por todos los profesores de lengua. En consecuencia, esto potenciaría la homologación de los procesos dentro de la pedagogía y generaría un proceso colectivo distintivo en el grupo docente.

Referente a la explicitación de las estrategias de retroalimentación, estas también debiesen darse a conocer a los alumnos, en etapas iniciales de los cursos de lengua, para que ellos estén conscientes del proceso de corrección durante todo el programa. Esto podría, eventualmente, bajar los niveles de ansiedad de los estudiantes, lo que permitiría una mejor identificación afectiva y comprensión de la retroalimentación entregada por los docentes.

La información obtenida en esta investigación, además, permite concluir que no existe, como práctica instalada y regular, la explicación de los profesores al inicio del curso o en momentos posteriores, sobre el uso que harán de esta importante estrategia de retroalimentación oral correctiva para favorecer la corrección del error.

Frente a la controversia surgida en relación con si las representaciones explícitas o implícitas constituyen sistemas distintos, o si existen etapas intermedias en la representación lingüística (Krashen, 1982; Paradis, 2004; Ellis, 2009 y Dienes y Perner, 1999), las respuestas de los estudiantes y profesores en este estudio permiten concluir que ambos tipos de retroalimentación forman un continuo de estrategias con un mismo propósito.

El análisis de estos resultados permite concluir que existe una tensión pedagógica entre las percepciones de los profesores con respecto al fenómeno de provisión de retroalimentación correctiva y las expectativas de los alumnos del programa de Pedagogía en Inglés en relación con este proceso. Esta tensión, causada principalmente por el desconocimiento de los alumnos acerca de la naturaleza de las diferentes estrategias de retroalimentación oral correctiva y los criterios que determinan su uso, por una parte, y por la irregularidad en el uso y frecuencia, por parte de los profesores, genera altos niveles de ansiedad de los aprendices. Ellos no serían capaces de percibir la intención correctiva de estas intervenciones pedagógicas.

Adicionalmente, la irregularidad de uso de determinadas estrategias de retroa- 
limentación oral correctiva percibida por los estudiantes incidiría en un efecto negativo progresivo en el proceso de adquisición de una segunda lengua, lo que podría potenciar un proceso de fosilización en un contexto instruccional en el que se espera que la corrección sea realizada por el profesor. Si bien este estudio no tiene como propósito intervenir la realidad y percepción que tienen profesores y alumnos sobre el uso de la retroalimentación en contextos oral de aprendizaje del idioma inglés, surge el interés del grupo de investigadores por contribuir a optimizar el proceso formativo profesional en la carrera que sirve de muestra a este estudio, compartiendo la información cualitativa y cuantitativa obtenida con el equipo directivo y docente que se desempeñan en el programa académico.

En resumen, esta investigación en el contexto universitario proporciona, en general, evidencia de problemas similares encontrados en estudios previos (Lyster y Ranta, 1997; Hernández y Reyes, 2012; Kirgoza y Agcamb, 2015), a saber, inconsistencia en el uso de estrategias de retroalimentación oral correctiva, retroalimentación correctiva no enfocada y no sistemática, miedo de corregir al alumno, incomprensión de la retroalimentación por parte del alumno por desconocimiento de las técnicas de retroalimentación oral correctiva y/o por bajo nivel lingüístico en la lengua meta, escaso uso de estrategias correctivas como recasts, mayor uso de técnicas de retroalimentación explícitas y escaso seguimiento a la corrección del error después de corregido.

\subsection{Implicaciones pedagógicas}

En el contexto de este estudio de las percepciones de profesores y alumnos de un programa de Pedagogía en Inglés, en una universidad estatal, en los tres niveles de creencias, a saber, conocimiento teórico y práctico de estrategias de retroalimentación correctiva, los criterios que determinan su uso y la frecuencia de uso de estas, el análisis cuantitativo y cualitativo de los resultados permite concluir que existe un nivel de divergencia entre las creencias de profesores y aprendices que podrían tener un efecto negativo en el proceso de adquisición de una segunda lengua. Para mejorar esta situación se sugiere la creación de cursos de inducción a las estrategias de retroalimentación oral correctiva, para estudiantes y profesores del programa de Pedagogía en Inglés a inicio de cada año lectivo.

Desde las conclusiones se desprende el hecho que los estudiantes del programa señalarían que no existe continuidad en el tratamiento del error, que se corrige por medio de estrategias correctivas orales en clase, pero que esta corrección no persistiría en el tiempo. Esta situación podría ser revertida por medio de la instalación de una práctica de retroalimentación oral correctiva al interior del programa que sea constante y sistemática y que se preocupe de seguir el uptake de los estudiantes. 


\section{REFERENCIAS}

Ammar, Ahlem y Spada, Nina. (2006). One size fits all? Recasts, prompts, and L2 learning. Studies in Second Language Acquisition, 28(4), 543-574.

Ayoun, Dalila. (2004). The effectiveness of written recast in the second language acquisition of aspectual distinction in French: A follow-up study. The Modern Language Journal, 88(1), 31-35.

Brown, Alan. (2009). Students' and teachers' perceptions of effective foreign language teaching: A comparison of ideals. The Modern Language Journal 93(1), 46-60.

Corder, Stephen Pit. (1981). Error analysis and interlanguage. Oxford: OUP.

Chaudron, Craig. (1988). Second language classrooms. Research on teaching and learning. Cambridge: Cambridge University Press.

DeKeyser, Robert. (1998). Beyond focus on form: Cognitive perspectives on learning and practicing second language grammar. En C. Doughty y J. Williams (Eds.), Focus on form in classroom second language acquisition (pp. 42-63). Cambridge, United Kingdom: Cambridge University Press.

DeKeyser, Robert. (2003). Implicit and explicit learning. En C. J. Doughty y M. H. Long (Eds.), The handbook of second language acquisition (pp. 313-348). Oxford, United Kingdom: Blackwell.

DeKeyser, Robert. (2007). Introduction: Situating the concept of practice. En R. DeKeyser (Ed.), Practice in a second language: Perspectives from applied linguistics and cognitive psychology (pp. 1-18). New York, United States: Cambridge University Press.

Dienes, Zoltan y Perner, Josef. (1999). A theory of implicit and explicit knowledge. Behavorial and Brain Sciences, 22, 735-808.

Ellis, Nick C. (2002). Frequency effects in language processing: A review with implications for theories of implicit and explicit language acquisition. Studies in Second Language Acquisition 24(2): 143-88.

Ellis, Nick. (2009). Language as a complex adaptive system. Language Learning 59(1), 1-26.

Ellis, Rod. (1993). Second language acquisition and the structural syllabus. TESOL Quarterly, 27(1), 91-113.

Ellis, Rod. (2009). A typology of written corrective feedback types. ELT Journal, 63(2), 97-107.

Ellis, Rod. (2011). Implicit and explicit SLA and their interface. En C. Sanz y R. Leow (Eds.), Implicit and explicit language learning: Conditions, processes, and knowledge in SLA and bilingualism (pp. 35-47). Washington, DC: Georgetown University Press.

Ellis, Rod y Youngee, Sheen. (2006). Reexamining the role of recasts in second language acquisition. Studies in Second Language Acquisition 28(4), 575-600. 
Ellis, Rod, Loewen, Shawn y Erlam, Rosemary. (2006). Implicit and explicit corrective feedback and the acquisition of L2 grammar. Studies in Second Language Acquisition, 28(2), 268-339.

Ferreira, Anita. (2006). Estrategias efectivas de feedback positivo y correctivo en el español como lengua extranjera. Signos, 39, 379-406.

Ferris, Dana. (2006). Does error feedback help student writers? New evidence on the short- and long-term effects of written error correction. En K. Hyland y F. Hyland (Eds.), Feedback in second language writing: Contexts and issues (pp. 81-104). Cambridge: Cambridge University Press.

Fujii, A., Ziegler, N., y Mackey, A. J. (2016). Peer interaction and metacognitive instruction in the EFL classroom. En M. Sato, y S. Ballinger (Eds.), Peer interaction and second language learning: Pedagogical potential and research agenda (pp. 63-89). Language learning and language teaching; Vol 45. John Benjamins.

Han, Zhaohong. (2002). A study of the impact of recasts on tense consistency in L2 output. TESOL Quarterly, 36, 543-572.

Hernández, Edith y Reyes, María del Rosario. (2012). Teachers' perceptions about oral corrective feedback and their practice in EFL classroom. PROFILE, 14(2), 63-75.

Jean, Gladys y Daphneé Simard. (2011). Grammar learning in English and French L2: Students' and teachers' beliefs and perceptions. Foreign Language Annals 44(4), 465-492.

Karmiloff-Smith, Annette. (1995). Beyond modularity: A developmental perspective on cognitive science. Cambridge. Mass.: MIT Press/Bradford Books.

Kirgoza Yasemin. y Agcamb, Reyhan. (2015). Teachers' perceptions on corrective feedback in Turkish primary schools. Procedia. Social and Behavioral Sciences 192, 574-581.

Krashen, Stephen. (1982). Principles and practice in second language acquisition. Oxford, Reino Unido: Pergamon.

Lasagabaster, David y Juan Sierra. (2005). Error correction: Students'versus teachers'perceptions. Language Awareness 14(2-3), 11-127.

Leeman, Jennifer. (2003). Recasts and second language development: Beyond negative evidence. Studies in Second Language Acquisition. 25(1), 37-63.

Long, M. H. (1996). The role of the linguistic environment in second language acquisition. En W. C. Ritchie y T. K. Bhatia (Eds.), Handbook of second language acquisition (pp. 413-468). New York: Academic Press.

Long, Michael. (2001). Focus on form. A feature in language teaching methodology. En C. Candlin y Merci, N. (Eds.), Second language teaching in its social context (pp. 413-454). London: Routledge.

Lyster, Roy. (2004). Differential effects of prompts and recasts in form-focused instruction. Studies in Second Language Acquisition. 26(3), 399-432. 
Lyster, Roy y Ranta, Leila. (1997). Corrective feedback and learner uptake: Negotiation of form in communicative classrooms. Studies in Second Language Acquisition, 19(1), 37-66.

Lyster, Roy y Saito, Kasuya. (2010). Oral feedback in classroom SLA: A metaanalysis. Studies in Second Language Acquisition 32(2), 265-302.

Lyster, Roy, Saito, Kazuya y Sato, Masatoshi. (2013). Oral corrective feedback in second language classrooms. Language Teaching, 46(1), 140 doi:10.1017/ S026144481200036

Mackey, Alison, Rhondal, Oliver, Leeman, Jennifer. (2003). Interactional input and the incorporation of feedback: An exploration of NS-NNS and NNSNNS adult and child dyads. Language Learning. 53(1), 35-66.

Nicholas, Howard, Lightbown, Patsy y Spada, Nina . (2001). Recasts as feedback to language learners. Language Learning, 51(4), 719-758.

Ortega, Lourdes. (2009). Understanding second language acquisition. London: Hodder Education.

Paradis, Michel. (1994). Neurolinguistic aspects of implicit and explicit memory: Implications for bilingualism and second language acquisition (Ed.), Implicit and explicit language learning (pp. 393-419). London: Academic Press.

Paradis, Michel. (2004). A neurolinguistic theory of bilingualism. Amsterdam: John Benjamins.

Pawlak, Miroslaw. (2014). Error correction in the foreign language classroom: Reconsidering the issues. Heidelberg/New York: Springer.

Philp, Jenefer. (2003). Constraints on "noticing the gap": Nonnative speakers' noticing of recasts in NS-NNS interaction. Studies in Second Language Acquisition, 25(1), 99-126.

Pienemann, M. (1989). Is language teachable? Psycholinguistic experiments and hypotheses. Applied Linguistics 10, 52-79.

Sato, Masatoshi. (2011). Constitution of form-orientation: Contributions of context and explicit knowledge to learning from recasts. Canadian Journal of Applied Linguistics, 14(1), 1-28.

Sato, Masatoshi y Lyster, Ranta. (2012). Peer interaction and corrective feedback for accuracy and fluency development. Studies in Second Language Acquisition. 34(4), 591-626.

Sato, Masatoshi y Loewen, Shawn. (2018). Metacognitive instruction enhances the effectiveness of corrective feedback: Variable effects of feedback types and linguistic. Language Learning 68(2), 507-545. Language Learning Research Club, University of Michigan.

Schachter, Jacquelyn. (1991). Corrective feedback in historical perspective. Second Language Research, 7(2), 89-102.

Schmidt, Richard y Frota, Sonya. (1986). Developing basic conversational ability in a second language: A case study of an adult learner of Portuguese. En R. 
R. Day (Ed.), Talking to learn: Conversation in second language acquisition (pp. 237-326). Rowley, MA: Newbury House.

Sheen, Youngee. (2004). Corrective feedback and learner uptake in communicative classroom across instructional contexts. Language Teaching Research, 8(1), 263-300.

Sheen, Youngee. (2011). Corrective feedback, individual differences and second language learning. Dordrecht: Springer.

Sheen, Youngee y Ellis, Rod. (2011). Corrective feedback in language teaching. En E. Hinkel (Ed.), Handbook of research in second language teaching and learning (pp. 593-610). New York: Routledge. 